\title{
Signal Processing Advances in Robots and Autonomy
}

\author{
Frank Ehlers, ${ }^{1}$ Fredrik Gustafsson (EURASIP Member), ${ }^{2}$ and Matthijs Spaan ${ }^{3}$ \\ ${ }^{1}$ NURC, NATO Research Centre, Viale S. Bartolomeo 400, 19126 La Spezia, Italy \\ ${ }^{2}$ Department of Electrical Engineering, Linköping University, 58183 Linköping, Sweden \\ ${ }^{3}$ Instituto de Sistemas e Robótica, Instituto Superior Técnico, Avenida Rovisco Pais 1, 1049-001 Lisboa, Portugal \\ Correspondence should be addressed to Frank Ehlers, frankehlers@ieee.org
}

Received 16 June 2009; Accepted 16 June 2009

Copyright ( 2009 Frank Ehlers et al. This is an open access article distributed under the Creative Commons Attribution License, which permits unrestricted use, distribution, and reproduction in any medium, provided the original work is properly cited.

The capabilities of robots and autonomous systems have increased dramatically over the past years. This success story partly depends on advances in signal processing which provide appropriate and efficient analysis of sensor data and enable autonomy.

A key element of the transition of signal processing output to its exploitation inside robots and autonomous systems is the way uncertainty is managed: uncertainty originating from insufficient sensor data, uncertainty about effects of future autonomous actions, and, in the case of distributed sensors and actuators (like for a team of robots), uncertainty about communication lines.

The aim of this special issue is to focus on recent developments that allow passing this transition path successfully, showing either where signal processing is used in robotics and autonomy or where robotics and autonomy had special demands that had not been fulfilled by signal processing before.

The articles in this special issue cover the following topics.

\section{Autonomous Navigation}

"Vector Field Driven Design for Lightweight Signal Processing and Control Schemes for Autonomous Robotic Navigation," "Vision-based Unmanned Aerial Vehicle Navigation Using Geo-referenced Information," "Automatic evaluation of landmarks for image based navigation update," and "PurePursuit Reactive Path Tracking for Non-Holonomic Mobile Robots with a 2D Laser-Scanner."

\section{Robot Teams and Exploration}

"Collaborative Area Monitoring Using Wireless Sensor Networks with Stationary and Mobile Nodes," and "A Common
Coordinates/Heading Direction Generation Method for a Robot Swarm with only RSSI-Based Ranging."

\section{Target Tracking Applications}

"Self-Localisation and Stream Field Based Partially Observable Moving Object Tracking," "A POMDP Framework for Coordinated Guidance of Autonomous UAVs for Multitarget Tracking," and "Prioritized Multi-Hypothesis Tracking by a Mobile Robot."

\section{Autonomous Navigation}

N. J. Mathai et al. address the problem of realizing lightweight signal processing and control architectures for agents in multirobot systems. They present the design of an analogamenable signal processing scheme. They use control and dynamical systems theory both as a description language and as a synthesis toolset to rigorously develop the computational machinery; these mechanisms are mated with structural insights from behavior-based robotics to compose overall algorithmic architectures. Their perspective is that robotic behaviors consist of actions taken by an agent to cause its sensory perception of the environment to evolve in a desired manner. To provide an intuitive aid for designing these behavioral primitives they present a novel visual tool, inspired vector field design, that helps the designer exploit the dynamics of the environment. They present simulation results and animation videos to demonstrate the signal processing and control architecture in action.

G. Conte et al. investigate the possibility of augmenting an Unmanned Aerial Vehicle (UAV) navigation system with a passive video camera in order to cope with long-term GPS outages. Their paper proposes a vision based navigation architecture which combines inertial sensors, visual 
odometry, and registration of the on-board video to a georeferenced aerial image. The vision-aided navigation system developed is capable of providing high-rate and drift-free state estimation for UAV autonomous navigation without the GPS system. Due to the use of image-to-map registration for absolute position calculation, drift-free position performance depends on the structural characteristics of the terrain.

Experimental evaluation of the approach based on offline flight data is provided. In addition, the architecture proposed has been implemented onboard as an experimental UAV helicopter platform and tested during vision-based autonomous flights.

S. Lang et al. address the automatic evaluation of landmarks for image-based navigation updates.

The successful mission of an autonomous airborne system like an unmanned aerial vehicle strongly depends on its accurate navigation. While GPS is not always available and pose estimation based solely on Inertial Measurement Unit drifts, image-based navigation may become a cheap and robust additional pose measurement device. For the actual navigation update they use a landmark-based approach. They found that it is essential that the used landmarks are well chosen. Therefore, they introduce an approach for evaluating landmarks in terms of the matching distance, which is the maximum misplacement in the position of the landmark that can be corrected. They validate the evaluations with a $3 \mathrm{D}$ reconstruction system working on data captured from a helicopter.

J. Morales et al. investigate the application of the Pure-Pursuit path tracking method for reactive tracking of paths that are implicitly defined by perceived environmental features. Due to its simplicity and efficiency, the Pure-Pursuit path tracking method has been widely employed for planned navigation of non-holonomic ground vehicles. Goal points are obtained through an efficient interpretation of range data from an onboard 2D laser-scanner to follow persons, corridors and walls. Moreover, this formulation allows that a robotic mission can be composed of a combination of different types of path segments. They have successfully tested these techniques in an indoor environment.

\section{Robot Teams and Exploration}

T. Lambrou et al. address the task of collaborative area monitoring using wireless sensor networks with stationary and mobile nodes. Monitoring a large area with stationary sensor networks requires a very large number of nodes which with current technology implies a prohibitive cost. The motivation of their work is to develop an architecture where a set of mobile sensors will collaborate with the stationary sensors in order to reliably detect and locate an event. The main idea of this collaborative architecture is that the mobile sensors should sample the areas that are least covered (monitored) by the stationary sensors. Furthermore, when stationary sensors have a "suspicion" that an event may have occurred, they report it to a mobile sensor that can move closer to the suspected area and can confirm whether the event has occurred or not. An important component of the proposed architecture is that the mobile nodes autonomously decide their path based on local information (their own beliefs and measurements as well as information collected from the stationary sensors in a neighborhood around them).

S. Hara et al. present a common coordinates/heading direction generation method for a robot swarm with only Received Signal Strength Indicator-based ranging. In the motion control of a microrobot swarm, a key issue is how to autonomously generate a set of common coordinates among all robots and to notify each robot of its heading direction in the generated common coordinates, without any special devices for estimating location and bearing. The authors propose a set of common coordinates and a heading direction generation method for a robot swarm with only Received Signal Strength Indicator (RSSI) measured through wireless communications. They explain the principle of the proposed method and show some computer simulation results on the location and direction estimation errors. Finally, experimental results demonstrate using a swarm composed of five robots with the IEEE 802.15.4 standard as its wireless communication tool.

\section{Target Tracking Applications}

K.-S. Tseng et al. present an algorithm for self-localization and stream field based partially observable moving object tracking. Self-localisation and object tracking are key technologies for human-robot interactions. Most previous tracking algorithms focus on how to correctly estimate the position, velocity, and acceleration of a moving object based on the prior state and sensor information. What has been rarely studied so far is how a robot can successfully track the partially observable moving object with laser range finders if there is no preanalysis of object trajectories. In this case, traditional tracking algorithms may lead to the divergent estimation. The authors introduce a novel laser range finder based partially observable moving object tracking and self-localization algorithm for interactive robot applications. Dissimilar to the previous work, they adopt a stream field-based motion model and combine it with the Rao-Blackwellised particle filter (RBPF) to predict the object goal directly. This algorithm can keep predicting the object position by inferring the interactive force between the object goal and environmental features when the moving object is unobservable. Experimental results show that the robot with the proposed algorithm can localize itself and track the frequently occluded object. Compared with the traditional Kalman filter and particle filter based algorithms, the proposed one significantly improves the tracking accuracy.

S. Miller et al. discuss the application of the theory of partially observable Markov decision processes (POMDPs) to the design of guidance algorithms for controlling the motion of unmanned aerial vehicles with onboard sensors to improve tracking of multiple ground targets. While POMDP problems are intractable to solve exactly, principled approximation methods can be devised based on the theory that characterizes optimal solutions. A new approximation method called nominal belief-state optimization (NBO), 
combined with other application-specific approximations and techniques within the POMDP framework, produces a practical design that coordinates the UAVs to achieve good long-term mean-squared-error tracking performance in the presence of occlusions and dynamic constraints. The flexibility of the design is demonstrated by extending the objective to reduce the probability of a track swap in ambiguous situations.

P. Rybskie et al. apply prioritized multihypothesis tracking to state estimation tasks of a mobile robot.

To act intelligently in complex and dynamic environments, mobile robots must estimate the position of objects by using information obtained from a wide variety of sources. The authors formally describe the problem of estimating the state of objects in the environment where the robot can only task its sensors to view on object at a time. They contribute an object tracking method that generates and maintains multiple hypotheses that consist of a probabilistic state estimate that is generated by the individual sources of information. These different hypotheses can be spatially disjoint such that they cannot all be viewed/verified by robot's sensors simultaneously. Thus, the robot must decide toward which hypothesis its sensors should be tasked by evaluating each hypothesis on its likelihood of containing the object. The rankings of these hypotheses are set by the expected uncertainty in the object's motion/process model, as well as the uncertainties in the sources of information used to track their positions. A detailed description of the algorithm is given together with extensive empirical results in simulation as well as experiments on actual robots that demonstrate the effectiveness of the approach taken.

\section{Acknowledgment}

The guest editors of this special issue are much indebted to their authors and reviewers, who put a tremendous amount of effort and dedication to make this issue a reality.

Frank Ehlers

Fredrik Gustafsson Matthijs Spaan 